\title{
LDL-C Targets in Secondary Prevention: How Low Should We Go?
}

\section{Karim Bayoumy ${ }^{1} \cdot$ Mohammed Gaber $^{1} \cdot$ Preethi Mani $^{2} \cdot$ Rishi Puri $^{2} \cdot$ Eoin Donnellan ${ }^{2} \cdot$ Leslie Cho $^{2} \cdot$ Donald Clark III $^{3}$. Seth S. Martin ${ }^{4}$ - Mohamed B. Elshazly ${ }^{1,5}$}

Published online: 18 June 2019

(C) The Author(s) 2019

\begin{abstract}
Purpose of Review The benefits of lowering low-density lipoprotein cholesterol (LDL-C), mainly using high-intensity statin therapy, and its impact on decreasing the recurrence of atherosclerotic cardiovascular disease (ASCVD) in secondary prevention has been well established. With the advent of non-statin medications, particularly PCSK-9 inhibitors, which can lower LDL-C to very low levels not seen before, it is important to answer some important questions regarding LDL-C lowering and the uses of these medications in clinical practice: how low should we go with LDL-C reduction? Is there a threshold beyond which lower LDL-C is not associated with any benefit and possibly harm? Does the benefit derived from more aggressive LDL-C lowering justify the cost of additional therapies?

Recent Findings Our review has found overwhelming evidence to support the conclusion that lower achieved LDL-C levels correlate with a decreased burden of atherosclerosis and better clinical outcomes in secondary prevention. The concern for adverse effects with very low LDL-C levels is not backed by the literature, and side effects appear to be medication-specific. There still remains a question of the cost-effectiveness of some non-statin therapies particularly PCSK9 inhibitors, in spite of recent price decreases, and whether the benefit is worth the cost.

Summary It is prudent to always pursue an individualized patient-level approach to LDL-C lowering that considers the patient's global cardiovascular risk, their side effect profile, and the cost-effectiveness of therapies in order to derive maximal benefit from aggressive lipid lowering.
\end{abstract}

Keywords Clinical guidelines $\cdot$ LDL lowering $\cdot$ Secondary prevention $\cdot$ PCSK9 inhibitors $\cdot$ Adverse effects

Karim Bayoumy and Mohammed Gaber are co-first authors.

This article is part of the Topical Collection on Lipids

Mohamed B. Elshazly

mes2015@qatar-med.cornell.edu

Karim Bayoumy

karimbayoumy@gmail.com

Mohammed Gaber

Dr.mgaber89@gmail.com

Preethi Mani

pmani87@gmail.com

Rishi Puri

rishi_puri@hotmail.com

Eoin Donnellan

DONNELE@ccf.org

Leslie Cho

chol@ccf.org
Donald Clark, III

dclark2@umc.edu

Seth S. Martin

smart100@jhmi.edu

Department of Medicine, Hamad Medical Corporation, Doha, Qatar

2 Department of Cardiovascular Medicine, Cleveland Clinic, Cleveland, OH, USA

3 Division of Cardiology, University of Mississippi Medical Center, Jackson, MS, USA

4 Department of Cardiovascular Medicine, Johns Hopkins Hospital, Baltimore, MD, USA

5 Department of Cardiovascular Medicine, Weill Cornell Medicine, Education City PO Box 24144, Doha, Qatar 


\section{Introduction}

The benefits of lowering low-density lipoprotein cholesterol (LDL-C), mainly using high-intensity statin therapy (HIST), and its impact on decreasing the recurrence of atherosclerotic cardiovascular disease (ASCVD) in secondary prevention has been well established. In the last few years, several large-scale trials using non-statin LDL lowering therapies have also shown significant reduction in ASCVD. As such, the most recent $2018 \mathrm{AHA} / \mathrm{ACC} / \mathrm{Multi}$-Society guideline, in concordance with other worldwide guidelines, has shifted towards recommending HIST with a goal of $\geq 50 \%$ LDL-C reduction with consideration of adding non-statin therapies if LDL-C level remains $\geq 70 \mathrm{mg} / \mathrm{dL}$ or non-HDL-C level $\geq 100 \mathrm{mg} / \mathrm{dL}$ in an incremental fashion, particularly in very high-risk individuals $[1 \bullet \bullet, 2 \bullet \bullet$.

With the advent of non-statin medications, particularly proprotein convertase subtilsin-kexin type 9 inhibitors (PCSK9i), which can lower LDL-C to very low levels not seen before, it is important to answer some important questions regarding LDL-C lowering and the uses of these medications in clinical practice: how low should we go with LDL$\mathrm{C}$ reduction? Is there a threshold beyond which lower LDL-C is not associated with any benefit and possibly harm? Does the benefit derived from more aggressive LDL-C lowering justify the cost of additional therapies? To answer these timely and crucial questions, we must review basic concepts of LDL-C lowering for ASCVD risk reduction and analyze the safety of very low LDL-C levels, as well as the cost-effectiveness of adding non-statin therapies.

\section{Evidence for Lower Is Better}

The formation and growth of atheromatous plaques, which underlie the pathophysiology of ASCVD, has been shown to be a consequence of circulating atherogenic apolipoproteins, mostly in the form of LDL particles. It has been argued that, when the lifetime burden of LDL-C measured by $\mathrm{mg} / \mathrm{dL}$ years (age x [LDL-C] reaches a threshold of $5000 \mathrm{mg} / \mathrm{dL}$-years, the accumulated atheroma begins to manifest as clinically overt ASCVD. Beyond this threshold, the risk of developing ASCVD increases logarithmically $[3 \bullet, 4 \bullet]$.

Mendelian randomization studies have been referred to as a "natural randomization" for LDL-C levels [5, 6], revealing that those with genetically lower LDL-C levels have a resulting lower risk for ASCVD. Using a Mendelian randomization approach, over 112,000 individuals from 14 prospective cohorts were analyzed. Those with higher proprotein convertase subtilsin-kexin type 9 (PCSK9) or 3-hydroxy-3methyl-glutaryl-coenzyme A reductase (HMGCR) scores, favoring lower LDL-C, had a $19 \%$ lower risk of myocardial infarction (MI) or death from coronary heart disease (CHD) per $10 \mathrm{mg} / \mathrm{dL}$ decrement in LDL-C. This effect was seen to be additive in a $2 \times 2$ factorial analysis of PCSK9 and HMGCR genetic scores $[7 \bullet \bullet]$. These findings were also seen in Niemann-Pick C1-Like 1 (NPC1L1) variants, the target mechanism in ezetimibe, who have low LDL-C. These studies suggest that genetically determined lifelong lower LDL-C levels are associated with a significantly reduced ASCVD risk, regardless of the underlying mechanism [8••].

Extending from the studies examining genetically determined LDL-C and cardiovascular risk, several meta-analyses have demonstrated a similar dose-dependent relationship between the lowering of LDL-C and decreased risk of ASCVD. The landmark meta-analysis by the Cholesterol Treatment Trialists' (CTT) Collaboration, examining the effect of LDL$\mathrm{C}$ lowering via statin on risk of major vascular events (coronary death, non-fatal MI, coronary revascularization, or stroke), revealed a relative risk reduction of $22 \%$ for every $1 \mathrm{mmol} / \mathrm{L}(38.7 \mathrm{mg} / \mathrm{dL})$ decrease in LDL-C [9]. More intensive statin therapy was beneficial, even if LDL-C was lower than $77 \mathrm{mg} / \mathrm{dL}$, with a resulting relative risk reduction of $20 \%$ per $38.7 \mathrm{mg} / \mathrm{dL}$ for CHD, and $16 \%$ for cardiac death. More recently, Navarese et al. performed a meta-analysis that included 34 primary and secondary prevention studies examining statin and non-statin therapies [10]. More intensive LDL$\mathrm{C}$ lowering was associated with a greater reduction in the risk of all-cause and cardiovascular mortality, but this effect did not extend to patients with baseline LDL-C $<100 \mathrm{mg} / \mathrm{dL}$ [10]. However, there continued to be a relative risk reduction of $10 \%$ in non-fatal MI, revascularization, and major adverse cardiovascular events (MACEs) in patients with baseline LDL-C $<100 \mathrm{mg} / \mathrm{dL}[10]$.

Another recent secondary prevention meta-analysis by Sabatine et al., which expanded on the CTT studies, further examined 3 studies of non-statins, and extrapolated a similar $21 \%$ relative risk reduction per $1 \mathrm{mmol} / \mathrm{L}(38.7 \mathrm{mg} / \mathrm{dL})$ reduction in LDL-C even for a group of patients starting with median LDL-C levels of $1.6 \mathrm{mmol} / \mathrm{L}(63 \mathrm{mg} / \mathrm{dL})$ and achieving ontreatment levels of $0.5 \mathrm{mmol} / \mathrm{L}(21 \mathrm{mg} / \mathrm{dL})[11 \bullet \cdot]$. In summary, there is overwhelming evidence that LDL-C is causal in atherosclerosis and that the lower the LDL-C level, the lower the risk of recurrent ASCVD in secondary prevention. Individuals with lifelong genetically-determined low LDL-C levels achieve approximately fourfold the amount of ASCVD risk reduction per LDL-C decrement compared to those with low LDL-C on drug therapy. Finally, although greater absolute risk reduction is derived from patients with higher baseline LDL-C ( $>100 \mathrm{mg} / \mathrm{dL}$ ), there is consistent relative risk reduction in patients with very low baseline LDL-C $<70 \mathrm{mg} / \mathrm{dL}$.

In addition to clinical outcome studies, serial coronary intravascular ultrasound (IVUS) imaging has allowed us to directly observe the effects of lipid lowering strategies on coronary atherosclerosis and establish a link between lower LDL$\mathrm{C}$ levels and changes in atheroma volume. IVUS studies have shown that in patients with CHD, coronary atheroma 
progression slows with statin therapy and even regresses with HIST. Lower LDL-C levels were associated with greater atheroma regression in several IVUS trials where patients were treated with HIST alone or in combination with non-statin therapies [12-14].

In the PRECISE-IVUS study, there was greater regression in atheromatous plaque with atorvastatin and ezetimibe combination therapy versus atorvastatin alone, corresponding with the lower LDL-C levels achieved with combination therapy [15]. The GLAGOV trial also demonstrated that patients with CHD on HIST had greater percent and total atheroma volume reduction with evolocumab compared with placebo, where the evolocumab group achieved lower mean, time-weighted average LDL-C levels (36.6 vs. $93.0 \mathrm{mg} / \mathrm{dL}$; difference, $56.5 \mathrm{mg} / \mathrm{dL}$ [95\% CI, -59.7 to -53.4$] ; P<.001)$ [16]. Serial coronary IVUS trials have thus added further mechanistic evidence to clinical outcome trials that LDL-C levels are causal in the process of atherosclerosis, and the lower the achieved LDL-C levels the better, when using statins and non-statin therapies that upregulate LDL receptor expression.

\section{Practical Application of "the Lower the Better" Approach to Individual Patients}

It is important to note that despite the decrease in relative risk that can be achieved with LDL-C lowering therapies; there will still continue to be the question of whether this translates to a meaningful reduction in absolute risk. The absolute risk reduction in ASCVD is more appreciable in those with a higher baseline ASCVD risk, higher baseline LDL-C level, and those who achieve a greater absolute reduction in LDL$\mathrm{C}$ with therapy. In Fig. 1, we demonstrate an example where we assume that 2 patients each has a similar global ASCVD risk of $25 \%$. Patient 1 has a baseline LDL-C level of $100 \mathrm{mg} /$ $\mathrm{dL}$ on HIST while patient 2 has a baseline LDL-C level of $70 \mathrm{mg} / \mathrm{dL}$ on HIST [11••]. Assuming both are treated with a PCSK9i, they will achieve $60 \%$ reduction in LDL-C levels. Patient 1 has a higher baseline LDL-C, and thus has a greater absolute reduction in LDL-C which results in a higher relative and absolute risk reduction. In Fig. 2, we show an example where patients 1 and 2 have different global ASCVD risk prior to treatment (patient $1-25 \%$ and patient $2-10 \%$ ), but both have a similar baseline LDL-C level of $75 \mathrm{mg} / \mathrm{dL}$. Assuming both are treated with PSCK9i, they will achieve a $60 \%$ reduction in LDL-C levels and approximately $24 \%$ relative risk reduction in ASCVD events, extrapolated from CTT. However, the absolute risk reduction in patient 1 is $6 \%$ vs. only $2.4 \%$ in patient 2 . Therefore, when approaching each individual patient, it is important to think about whether further LDL-C lowering will achieve meaningful reduction in absolute ASCVD risk. This individualized approach is applicable even in patients who achieve an LDL-C level of < $70 \mathrm{mg} / \mathrm{dL}$ with HIST as there still remains a subset who continue to have an elevated residual risk $[18,19]$. Such risk could be related to discordantly elevated lipoproteins such as apolipoprotein B (ApoB) and lipoprotein(a), or could be related to other factors such as elevated high-sensitivity $\mathrm{C}$-reactive protein (hsCRP) and auto-immune diseases $[18,20]$. In such

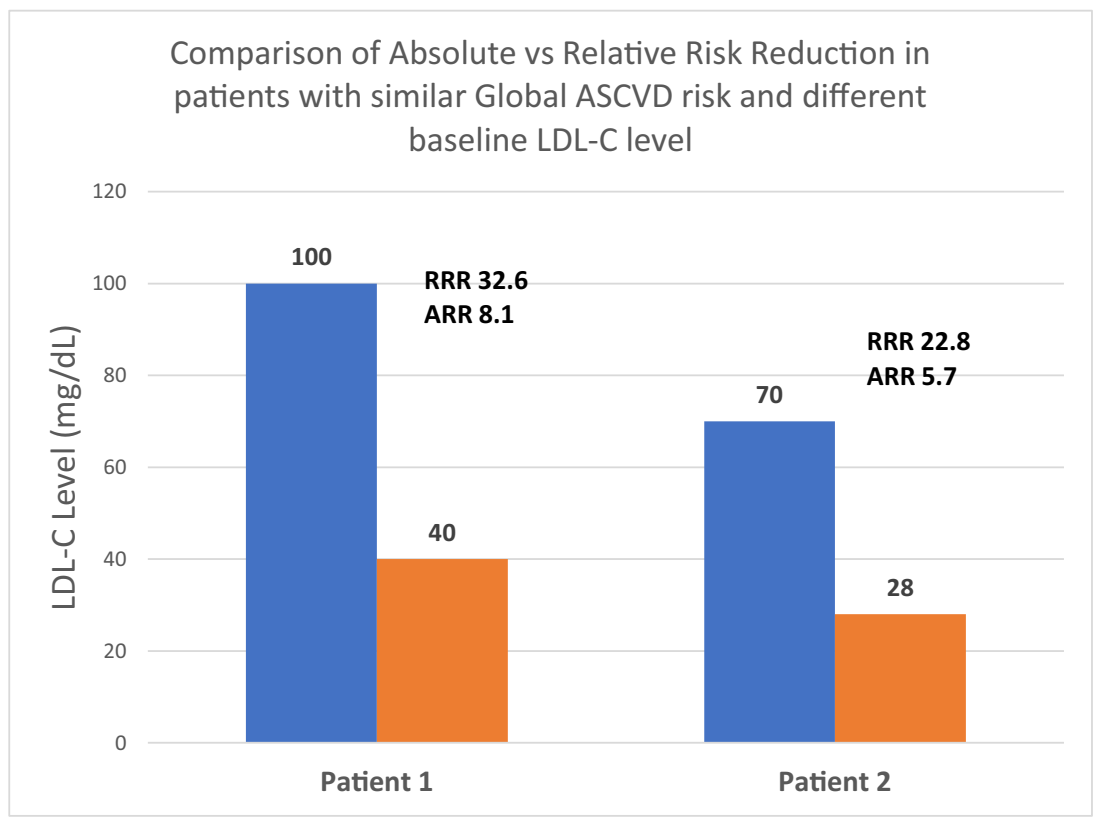

Fig. 1 Comparison of absolute risk reduction vs. relative risk reduction for two patients with a $25 \%$ global ASCVD risk, but different baseline LDL-C on HIST (patient $1-100 \mathrm{mg} / \mathrm{dL}$ vs. patient $2-70 \mathrm{mg} / \mathrm{dL}$ ). Assuming both patients have similar reduction in LDL-C by $60 \%$ with a PCSK9i, the relative risk reduction is substantial in both patients; however, the absolute risk reduction is less in the patient with lower baseline LDL-C [17] (based on findings of PCSK9i studies with short term follow-up). ARR absolute risk reduction, RRR relative risk reduction, HIST high-intensity statin therapy, PCSK9i proprotein convertase subtilsin-kexin type 9 inhibitor, ASCVD atherosclerotic cardiovascular disease 
Fig. 2 Comparison of absolute risk reduction in patients with different global ASCVD risk (patient $1-25 \%$ vs. patient 2$10 \%$ ), but the same baseline LDLC on HIST of $75 \mathrm{mg} / \mathrm{dL}$. Assuming both patients have a reduction in LDL-C by $60 \%$ with a PCSK9i, the relative risk reduction is equal in both patients (24\%); however, absolute risk reduction is much less in patient 2 $(2.4 \%)$ vs. patient $1(6 \%)$ (based on findings of PCSK9i studies with short term follow-up). ASCVD atherosclerotic cardiovascular disease, HIST high-intensity statin therapy, PCSK9i proprotein convertase subtilsin-kexin type 9 inhibitor
Comparison of Absolute vs Relative Risk

Reduction in patients with different global ASCVD risk and similar baseline LDL-C level

30

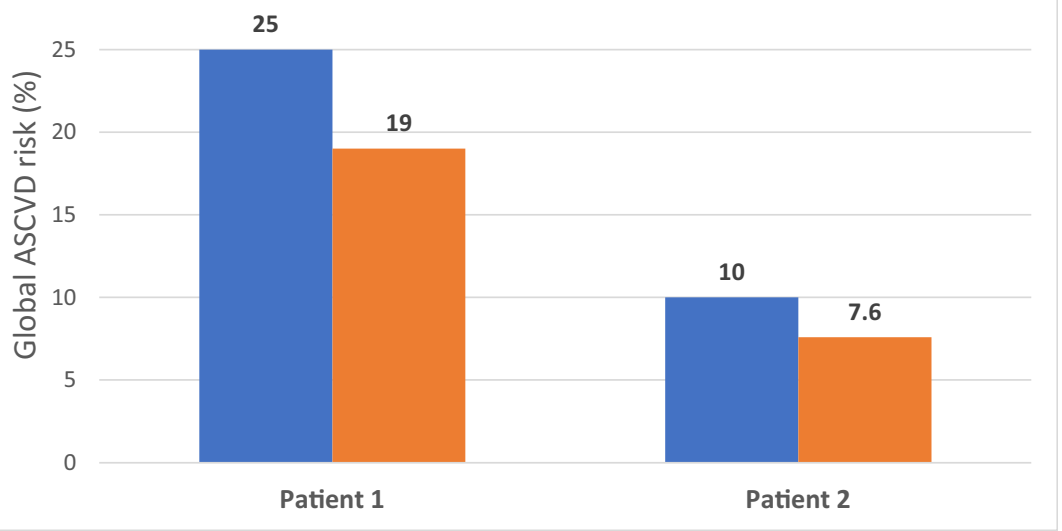

patients with elevated residual risk, more aggressive reduction in LDL-C levels, even beyond $70 \mathrm{mg} / \mathrm{dL}$, may translate into meaningful reduction in absolute ASCVD risk.

The 2018 AHA/ACC/Multi-Society Cholesterol guidelines have recommended an incremental approach to lowering LDL-C in secondary prevention. HIST is considered the mainstay of secondary prevention, with the expected decrease in LDL-C of approximately 50\% from baseline. Despite maximum tolerated statin therapy, many patients continue to have LDL-C levels $\geq 70 \mathrm{mg} / \mathrm{dL}$ [21]. In patients above this LDL-C threshold who have very high ASCVD risk, such as those with multiple recurrent events or other high-risk factors, the guideline recommends further lowering of LDL-C by adding ezetimibe to HIST, which can result in a $24 \%$ reduction in LDL-C as shown in the IMPROVE-IT trial [22••]. Even with the addition of ezetimibe, there still remains a subset of patients who are still above the LDL-C threshold of $\geq 70 \mathrm{mg} / \mathrm{dL}$ [21] and may be considered for treatment with PCSK9i. PSCK9i outcome trials, FOURIER and ODYSSEY Outcomes, showed that the addition of PCSK9i to HIST in secondary prevention patients was associated with a relative risk reduction of around 15\%; however, absolute risk reduction was $1.5-2 \%$ in this population. This has to be taken with caution because FOURIER followed patients for a median of only 2.2 years and the continued curve separation suggests that the treatment effect is expected to be larger on the long term. Therefore, it is imperative that clinicians take into consideration the patient's global ASCVD risk as well as the estimated absolute risk reduction before deciding to add non-statin therapies. Interestingly, an analysis of the FOURIER trial, which differentiated patients by their cardiovascular risk, noted a substantial difference in the absolute risk reduction derived by each group from therapy, ranging from >
$3 \%$ to approximately $1 \%$ in the lower risk groups [23]. For example, one may consider targeting lower LDL-C in patients with diabetes with multiple recurrent ASCVD events despite an achieved LDL-C level of $65 \mathrm{mg} / \mathrm{dL}$ on HIST, especially when the addition of non-statin therapies is cost-effective and not associated with adverse side effects.

\section{Adverse Effects of Very Low LDL-C Levels}

LDL-C lowering with statins has been previously linked with several adverse effects, including new-onset type 2 diabetes mellitus, hemorrhagic stroke, myositis, and neurocognitive side effects, some of which have been refuted. The European consensus panel on statins has noted a discordantly higher rate of statin-associated muscle symptoms (SAMS) (7-29\%) compared with RCTs [24]. Meanwhile, the STOMP randomized controlled trial showed a $9.4 \%$ rate of SAMS compared with $4.6 \%$ in controls [24]. However, the risk of myopathy was generally linked with the dose of statin, rather than the reduction of LDL-C achieved [24]. Moreover, the GAUSS-3 trial examined the use of ezetimibe or evolocumab monotherapy in patients with statin intolerance and showed that evolocumab was more efficient at reducing LDL-C in these patients and there were no significant SAMS reported [25].

On the other hand, an increased risk of type 2 diabetes mellitus has been reported in patients with very low LDL-C levels with conflicting evidence. In Mendelian randomization studies examining PCSK9i and HMGCR variants, those with a lower genetically determined LDL-C showed a respective $11.2 \%$ and $12.7 \%$ increase in the risk of diabetes, in a dosedependent and additive manner [7••]. On the other hand, a meta-analysis $[11 \bullet \cdot$ examining patients with very low LDL-C levels at baseline who were further treated with LDL-C 
lowering therapy showed no difference in the rate of new onset diabetes mellitus, cancer, or hemorrhagic stroke. In a pooled analysis of 10 studies from the ODYSSEY trials, which achieved a substantial decrease LDL-C levels as low as $30 \mathrm{mg} / \mathrm{dL}$, the incidence of diabetes was not increased [26••]. Regardless of this reported risk of new onset diabetes, an analysis from the JUPITER trial showed that the cardiovascular and mortality benefits of statin therapy exceed the diabetes hazard, including in participants at high risk of developing diabetes. Overall, for every 1 incident case of diabetes, 4 or 5 ASCVD events are prevented [27]. Similarly for hemorrhagic stroke, initial concerns were obviated by a large meta-analysis of 180,000 patients from randomized trials showing no increased risk and that there is a large net benefit with statins even in those at high risk of hemorrhagic stroke [28] Another meta-analysis evaluating the risk of hemorrhagic stroke with different LDL-C lowering therapies, showed no increased risk, even with metaregression of achieved LDL-C levels [29].

In a 2015 network meta-analysis of PCSK9i trials, there was an increased rate of neurocognitive adverse events with PCSK9i compared with placebo (OR 2.34 [95\% CI 1.114.93] $P=0.02$ ) [30]. However, the FOURIER trial, which examined the PCSK9i evolocumab compared to placebo for secondary prevention, showed no difference in the rate of neurocognitive effects [31]. In a subset of 1204 patients from the FOURIER study, neurocognitive testing was performed over a median follow up of 19.4 months comparing patients receiving PCSK9i vs. placebo on baseline HIST [32••]. The study revealed no association between adverse cognitive effects, even in those who achieved LDL-C $<25 \mathrm{mg} / \mathrm{dL}[32 \bullet \bullet]$. This study is limited by its relatively short duration and that both arms of the study were already on statin therapy. However, the results match those from Mendelian randomization studies that show no neurocognitive side effects of having genetically determined very low levels of LDL-C [33]. Further studies and careful post-marketing surveillance of PCSK9i on the long term will help further clarify if neurocognitive side effects are associated with very low levels of LDL-C. In summary, it seems that there is no evidence to suggest significant short- or long-term side effects that are directly associated with having very low LDL-C levels, and most reported side effects are usually medication specific.

\section{Cost-Effectiveness of LDL-C Lowering to Very Low Levels}

A model of 215,000 patients with MI from a US population receiving HIST with on-treatment LDL-C $\geq 70 \mathrm{mg} / \mathrm{dL}$ was conducted to determine the cost-effectiveness of alirocumab. The model was based on the inclusion criteria in the ODYSSEY trial, and compared to statin and statin vs. ezetimibe [34]. The authors assigned a cutoff of $\$ 100,000$ per quality-adjusted life year (QALY) to meet a willingness- to-pay threshold, which would only be feasible with $86 \%$ reduction of the price of alirocumab from its price of $\$ 14,560$ annually in March of 2018. The recent decrease in the price of alirocumab to $\$ 5850$ a year has been welcomed [35]; however, it still remains far from the recommended price of $\$ 874$ proposed by the authors if it is to be comparable to ezetimibe. The contention with alirocumab is adherence because only half of patients who are prescribed the drug are approved by insurance, with only a third ever receiving treatment. Furthermore, the patients who receive treatment are not always the ones most likely to benefit from treatment based on their risk [36]. Baum et al. have appraised the utility of costanalysis and identified that such analyses may rely on too many assumptions, including the acceptable cost goal, the baseline risk of a population, amongst others; without putting enough weight on the individual patient. Thus, while the benefits of extensive LDL-C lowering with non-statin therapies are apparent, their practical implementation is challenging and clinicians need to individually weigh the risks and benefits of additional therapies in each patient.

\section{Applying the "Lower the Better" Concept in Primary Prevention}

Primary prevention of ASCVD with LDL-C lowering therapies has mostly been recommended for those with high ASCVD risk scores. The exceptions are patients aged 40-75 years with diabetes mellitus (where at least a moderate-intensity statin is recommended regardless of 10-year risk score) and for those patients with severe primary hypercholesterolemia (where a high-intensity statin is recommended regardless of 10-year risk score). Risk scoring, which often weighs age as a strong predictor, is recommended for those without established risk factors $[2 \bullet \bullet]$. The CTT meta-analysis demonstrated that patients in the lower risk groups (5-year risk < $10 \%)$ also benefitted from more intensive statin therapy, with a relative risk reduction per $38.6 \mathrm{mg} / \mathrm{dL}$ of $43 \%$ in non-fatal MI and $48 \%$ in coronary revascularizations [37]. Following the CTT analysis, the HOPE-3 randomized controlled trial examined a diverse population of individuals without known ASCVD at moderate risk who were randomized to rosuvastatin $10 \mathrm{mg} /$ day vs. placebo [38]. Rosuvastatin monotherapy was associated with a decreased risk of composite death from ASCVD, non-fatal MI, or non-fatal stroke (hazard ratio, 0.76; 95\% CI, 0.64 to $0.91 ; P=0.002$ ) and a significant decrease in coronary revascularization, heart failure, and resuscitated cardiac arrest (hazard ratio, $0.75 ; 95 \% \mathrm{CI}$, 0.64 to $0.88 ; P<0.001)[39 \cdot \bullet$ ].

The results of these primary prevention trials as well as Mendelian randomized studies bring up the important concept of lifetime exposure to LDL-C. If the lifetime 
exposure to LDL-C is taken into consideration, the age of onset at which ASCVD begins may be significantly delayed [40]. This likely explains the increase in the observed risk reduction seen with LDL-C lowering therapies as the duration of treatment extends. In terms of primary prevention, the duration of LDL-C lowering would have to be extended significantly compared with secondary prevention trials, in order to establish a meaningful reduction in ASCVD risk [41]. An analysis from the Framingham Offspring cohort demonstrated that early treatment of moderately elevated lipid level may theoretically return a fourfold benefit over time, in comparison to starting treatment later [40]. However, there continues to be practical difficulties in conducting long-term studies of aggressive LDL lowering, given that a large segment of the population may qualify for early treatment, the duration of follow-up will likely be unfeasible, while the expected treatment effect may be small and difficult to power. But rather than being handicapped by skepticism, we should pursue creative and innovative approaches to answer several important questions related to primary prevention: Would individuals derive benefit from aggressive lifelong reduction in LDL-C starting in early adulthood? How low should we go with LDL-C and what tools should we use? Is lowering LDL-C, at a very young age, cost-effective and safe, especially with novel longterm interventions like gene therapy or RNA interference?

\section{Conclusion}

We are in an era of emerging LDL-C lowering therapies. Combining an armamentarium of tools can potentially achieve dramatic reductions in LDL-C levels and subsequently reduce the risk for ASCVD events. The concern for adverse effects with such low LDL-C levels has been raised; however, clinical studies thus far have been reassuring and side effects appear to be medication-specific. There still remains a question of the costeffectiveness of some non-statin therapies particularly PCSK9i, in spite of recent price decreases, and whether the benefit is worth the cost. Therefore, it is prudent to always pursue an individualized patient-level approach to LDL-C lowering that considers the patient's global cardiovascular risk, their side effect profile, and the cost-effectiveness of therapies in order to derive maximal benefit from aggressive lipid lowering.

Funding Information Open Access funding provided by the Qatar National Library.

\section{Compliance with Ethical Standards}

Conflict of Interest DCIII reports has received grant support from the American Heart Association. SSM has served on scientific advisory boards of Amgen, Sanofi, Regeneron, Esperion, Novo Nordisk, Quest Diagnostics, and Akcea Therapeutics, and has received research support from Apple, Google, iHealth, Nokia, NIH, the Maryland Innovation Initiative, American Heart Association, Aetna Foundation, PJ Schafer Memorial Fund, and David and June Trone Family Foundation. He is a co-inventor on a pending patent filed by Johns Hopkins University for a system of LDL-C estimation.

All other authors report no relevant financial disclosures.

Human and Animal Rights and Informed Consent All reported studies/ experiments with human or animal subjects performed by the authors have been previously published and complied with all applicable ethical standards (including the Helsinki declaration and its amendments, institutional/national research committee standards, and international/national/institutional guidelines).

Open Access This article is distributed under the terms of the Creative Commons Attribution 4.0 International License (http:// creativecommons.org/licenses/by/4.0/), which permits unrestricted use, distribution, and reproduction in any medium, provided you give appropriate credit to the original author(s) and the source, provide a link to the Creative Commons license, and indicate if changes were made.

\section{References}

Papers of particular interest, published recently, have been highlighted as:

- Of importance

•- Of major importance

1.• Catapano AL, Graham I, De Backer G, et al. 2016 ESC/EAS guidelines for the management of dyslipidaemias. Eur Heart J. 2016;37: 2999-3058. The most recent European guidelines on the management of dyslipidemia.

2.• Grundy SM, Stone NJ, Bailey AL, et al. 2018 AHA/ACC/ AACVPR/AAPA/ABC/ACPM/ADA/AGS/APhA/ASPC/NLA/ PCNA Guideline on the Management of Blood Cholesterol. Circulation. Updated guidelines but the American College of Cardiology for management of dyslipidemia.

3. Ference BA, Graham I, Tokgozoglu L, Catapano AL. Reprint of: impact of lipids on cardiovascular health. J Am Coll Cardiol. 2018;72:2980-95. A comprehensive review indicating the impact of lifetime exposure to LDL.

4. Ference BA. Mendelian randomization studies: using naturally randomized genetic data to fill evidence gaps. Curr Opin Lipidol. 2015;26:566-71.

5. Hingorani A, Humphries S. Nature's randomised trials. Lancet. 2005;366:1906-8.

6. Ference BA. How to use Mendelian randomization to anticipate the results of randomized trials. Eur Heart J. 2018;39:360-2.

7.• Ference BA, Robinson JG, Brook RD, Catapano AL, Chapman MJ, Neff DR, et al. Variation in PCSK9 and HMGCR and risk of cardiovascular disease and diabetes. N Engl J Med. 2016;375: 2144-53. Mendelian study showing the impact of genetically determined LDL levels and their impact on the risk for cardiovascular events.

8.• Ference BA, Majeed F, Penumetcha R, Flack JM, Brook RD. Effect of naturally random allocation to lower low-density lipoprotein cholesterol on the risk of coronary heart disease mediated by polymorphisms in NPC1L1, HMGCR, or both. J Am Coll Cardiol. 2015;65:1552-61. Mendelian study showing the impact of 
genetically determined LDL levels and their impact on the risk for cardiovascular events.

9. Cholesterol Treatment Trialists' (CTT) Collaboration, Baigent C, Blackwell L, et al. Efficacy and safety of more intensive lowering of LDL cholesterol: a meta-analysis of data from 170,000 participants in 26 randomised trials. Lancet. 2010;376:1670-81.

10. Navarese EP, Robinson JG, Kowalewski M, Kolodziejczak M, Andreotti F, Bliden K, et al. Association between baseline LDL-C level and total and cardiovascular mortality after LDL-C lowering. JAMA. 2018:319:1566-79.

11.• Sabatine MS, Wiviott SD, Im K, Murphy SA, Giugliano RP. Efficacy and safety of further lowering of low-density lipoprotein cholesterol in patients starting with very low levels. JAMA Cardiol. 2018;3:823. Meta-analysis of the utility and safety of more intensive LDL lowering therapy.

12. Kataoka Y, Andrews J, Puri R, Psaltis PJ, Nicholls SJ. Plaque burden, microstructures and compositions underachieving very low LDL-C levels. Curr Opin Endocrinol Diabetes Obes. 2017;24:122-32.

13. Elshazly MB, Stegman B, Puri R. Regression of coronary atheroma with statin therapy. Curr Opin Endocrinol Diabetes Obes. 2016;23: $131-7$.

14. Nicholls S, Ballantyne C, Barter P, et al. Effect of two intensive statin regimens on progression of coronary disease. Obes Metab. 2011:49.

15. Tsujita K, Sugiyama S, Sumida H, Shimomura H, Yamashita T, Yamanaga K, et al. Impact of dual lipid-lowering strategy with ezetimibe and atorvastatin on coronary plaque regression in patients with percutaneous coronary intervention. J Am Coll Cardiol. 2015;66:495-507.

16. Nicholls SJ, Puri R, Anderson T, Ballantyne CM, Cho L, Kastelein JJP, et al. Effect of evolocumab on progression of coronary disease in statin-treated patients. JAMA. 2016;316:2373.

17. Schmidt AF, Pearce LS, Wilkins JT, Overington JP, Hingorani AD, Casas JP. PCSK9 monoclonal antibodies for the primary and secondary prevention of cardiovascular disease. Cochrane Database Syst Rev. 2017(4):CD011748.

18. Khera AV, Everett BM, Caulfield MP, Hantash FM, Wohlgemuth J, Ridker PM, et al. Lipoprotein(a) concentrations, rosuvastatin therapy, and residual vascular risk. Circulation. 2014;129:635-42.

19. Willeit P, Ridker PM, Nestel PJ, Simes J, Tonkin AM, Pedersen TR, et al. Baseline and on-statin treatment lipoprotein(a) levels for prediction of cardiovascular events: individual patient-data meta-analysis of statin outcome trials. Lancet. 2018;392:1311-20.

20. O'Donoghue ML, Fazio S, Giugliano RP, Stroes ESG, Kanevsky E, Gouni-Berthold I, et al. Lipoprotein(a), PCSK9 inhibition, and cardiovascular risk. Circulation. 2019;139:1483-92.

21. Cannon CP, Khan I, Klimchak AC, Reynolds MR, Sanchez RJ, Sasiela WJ. Simulation of lipid-lowering therapy intensification in a population with atherosclerotic cardiovascular disease. JAMA Cardiol. 2017;2:959-66.

22.• Cannon CP, Blazing MA, Giugliano RP, McCagg A, White JA, Theroux P, et al. Ezetimibe added to statin therapy after acute coronary syndromes. N Engl J Med. 2015;372:2387-97. IMPROVEIT trial showing ezetimibe as viable LDL lowering therapy.

23. Sabatine MS, De Ferrari GM, Giugliano RP, et al. Clinical benefit of evolocumab by severity and extent of coronary artery disease. Circulation. 2018;138:756-66.

24. Stroes ES, Thompson PD, Corsini A, Vladutiu GD, Raal FJ, Ray KK, et al. Statin-associated muscle symptoms: impact on statin therapy-European Atherosclerosis Society Consensus Panel Statement on Assessment, Aetiology and Management. Eur Heart J. 2015;36:1012-22.

25. Nissen SE, Stroes E, Dent-Acosta RE, Rosenson RS, Lehman SJ, Sattar N, et al. Efficacy and tolerability of evolocumab vs ezetimibe in patients with muscle-related statin intolerance. JAMA. 2016;315: $1580-90$
26.• Colhoun HM, Ginsberg HN, Robinson JG, Leiter LA, MüllerWieland D, Henry RR, et al. No effect of PCSK9 inhibitor alirocumab on the incidence of diabetes in a pooled analysis from 10 ODYSSEY Phase 3 studies. Eur Heart J. 2016;37:2981-9. Meta-analysis focused on determining the impact of LDL lowering with PCSK9i and incident diabetes.

27. Ridker PM, Pradhan A, MacFadyen JG, Libby P, Glynn RJ. Cardiovascular benefits and diabetes risks of statin therapy in primary prevention: an analysis from the JUPITER trial. Lancet. 2012;380:565-71.

28. McKinney JS, Kostis WJ. Statin therapy and the risk of intracerebral hemorrhage. Stroke. 2012;43:2149-56.

29. Judge C, Ruttledge S, Costello M, et al. Lipid lowering therapy, low-density lipoprotein level and risk of intracerebral hemorrhagea meta-analysis. J Stroke Cerebrovasc Dis. 2019:1-7.

30. Lipinski MJ, Benedetto U, Escarcega RO, Biondi-Zoccai G, Lhermusier T, Baker NC, et al. The impact of proprotein convertase subtilisin-kexin type 9 serine protease inhibitors on lipid levels and outcomes in patients with primary hypercholesterolaemia: a network meta-analysis. Eur Heart J. 2016;37:536-45.

31. Sabatine MS, Giugliano RP, Keech AC, Honarpour N, Wiviott SD, Murphy SA, et al. Evolocumab and clinical outcomes in patients with cardiovascular disease. N Engl J Med. 2017;376:1713-22.

32.• Giugliano RP, Mach F, Zavitz K, Kurtz C, Im K, Kanevsky E, et al. Cognitive function in a randomized trial of evolocumab. N Engl J Med. 2017;377:633-43. A direct examination of neurocognitive function in patients in a RCT receiving PCSK9i.

33. Safarova MS, Satterfield BA, Fan X, et al. A phenome-wide association study to discover pleiotropic effects of PCSK9, APOB, and LDLR. NPJ Genom Med. 4.

34. Kazi DS, Penko J, Coxson PG, Guzman D, Wei PC, BibbinsDomingo K. Cost-effectiveness of alirocumab. Ann Intern Med. 2019;170:221.

35. Kuchler H. Sanofi and Regeneron cut price of Praluent by $60 \%$. Financ Times.

36. Navar AM, Taylor B, Mulder H, Fievitz E, Monda KL, Fievitz A, et al. Association of prior authorization and out-of-pocket costs with patient access to PCSK9 inhibitor therapy. JAMA Cardiol. 2017;2: 1217-25.

37. Mihaylova B, Emberson J, Blackwell L, et al. The effects of lowering LDL cholesterol with statin therapy in people at low risk of vascular disease: meta-analysis of individual data from 27 randomised trials. Lancet. 2012(380):581-90.

38. Lonn E, Bosch J, Pogue J, Avezum A, Chazova I, Dans A, et al. Novel approaches in primary cardiovascular disease prevention: the HOPE-3 trial rationale, design, and participants' baseline characteristics. Can J Cardiol. 2016;32:311-8.

39.• Yusuf S, Bosch J, Dagenais G, Zhu J, Xavier D, Liu L, et al. Cholesterol lowering in intermediate-risk persons without cardiovascular disease. N Engl J Med. 2016;374:2021-31. Recent HOPE-3 trial for primary prevention in an intermediate risk population.

40. Navar-Boggan AM, Peterson ED, D'Agostino RB, Neely B, Sniderman AD, Pencina MJ. Hyperlipidemia in early adulthood increases long-term risk of coronary heart disease. Circulation. 2015;131:451-8.

41. Sabatine MS. PCSK9 inhibitors: clinical evidence and implementation. Nat Rev Cardiol. 2019;16:155-65.

Publisher's Note Springer Nature remains neutral with regard to jurisdictional claims in published maps and institutional affiliations. 